\title{
A CORRELATION ANALYSIS BETWEEN FOUR ENERGY FIELD SCANNING DEVICES AND CONSCIOUS PERCEPTION OF BODILY ISSUES
}

Mark Abadi, Thornton Streeter and Daniel Ulanowsky The Centre for Human Energy Field Research, Exeter

\section{INTRODUCTION}

The medical community is continually seeking new methods to better understand the mechanism of disease. One area of increased interest is the study of the electromagnetic fields generated by the body as a measure of internal physical and mental health state. Medical analysis has long used information gleaned from electrical activity of the body to aid in diagnosis, for example Electro Encephalography (EEG). There is some suggestion that field measurements taken outside the body are often more indicative of events taking place within the body than are standard electrical measurements taken at the skin surface (Oschmann, 2000).

This study employs four commercially available technologies (GDV, ESM, PIP \& RFI) that are currently used by researchers and practitioners to measure the 'energetic' parameters of clients. Each of the devices has evidence from case studies, that has been able to demonstrate the possibility of identifying areas of physical and mental imbalance from scans. To date however only GDV has published research in peer review journals (Bundzen et al 2002 and Dobson et al 2000). This is the first study of its kind investigating the relationship between the results from four different scanners.

\section{MATERIALS AND METHODS}

The four devices comprise: Gas Discharge Visualisation (GDV), Electro Scanning Method (ESM), Polycontrast Interference Photography (PIP) and Resonant Field Imaging (RFI). Each device delivers the information in different formats. Both RFI and ESM provide raw data (frequencies), which in the case of RFI is then used to build up an image, whereas in ESM no computer software is involved and the raw data is used in analysis. With PIP, the process of data collection and image creation is automated GDV builds up an image based on readings taken at the fingertips

10 participants were chance selected; each requested to complete a body perception questionnaire, in which they identified current and past health issues. The four scanning sessions for each participant took place on the same day and in the same order. The scans were interpreted by experts using qualitative analysis and compared to the health data from the client questionnaire.

\section{RESULTS}

The results of this pilot study would seem to indicate a link between the energy fields identified by these scanning technologies and client health state. Of the four devices used each showed some capacity to detect imbalances around sites of known physical imbalance.

Another aspect of this study was exploring the validity of each device by comparison with each other. The analysis proved difficult and the results were mixed. Relationships were identified with PIP, RFI and GDV although less so with ESM. In some part, this could be due to the specific scanning pattern conducted with ESM as observation of improved correspondence was seen when scanning the same 17 sites as RFI analysis.

\section{Gas Discharge Visualisation (GDV)}

Technical difficulties were experienced with the GDV system, resulting in only four cases scanned. A clear relationship was indicated between overall health state perceived by the client and the observed energy states in the GDV aura scans. Furthermore GDV demonstrated some ability to correlate specific sites of imbalance.

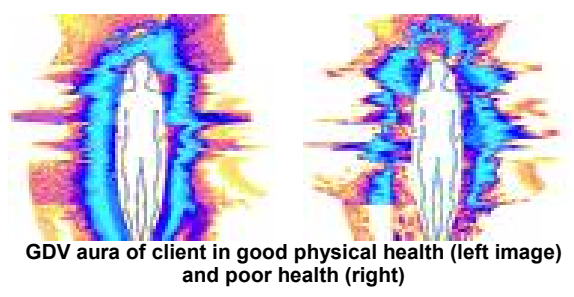

Electro Scanning Method (ESM)

ESM indicated some degree of imbalance in regions scanned, although deviations were slight and thus created difficulty in identifying significant associations. Only case studies 1 and 5 , in which all but two regions were identified as balanced could a significant association be identified. Greater relationship was identified when scanning pattern followed RFI model (17 body regions)

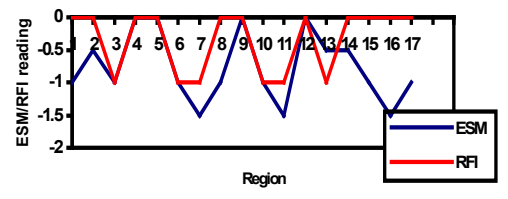

ESM and RFI readings of Case Study 2 at 17 body sites

Polycontrast Interference Photography (PIP)

As with GDV, PIP provides good association between overall health state and the energy field scan.
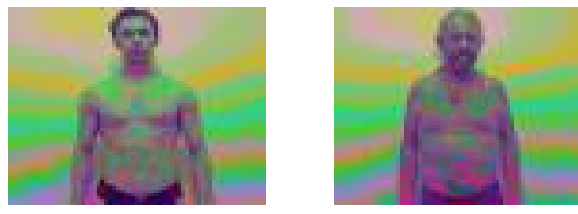

PIP scans: client in generally good health (left scan) and poorer health (right)
Furthermore some good identification of specific issues for examples knee pain in arthritis case (case study 10) and knee pain (case study 6) can be clearly seen.

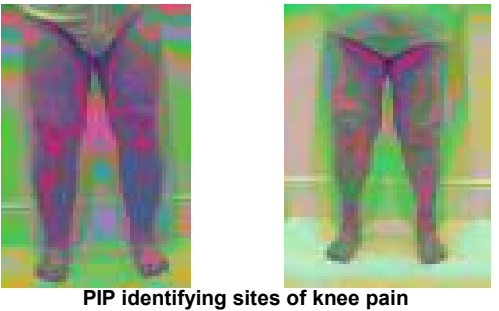

Resonant Field Imaging (RFI)

The relationship was evident between $\mathrm{RFI}$, client perception and the other devices. However there was some conflict where one client thought to be in good physical health did not appear so with RFI analysis as the images show below. Congestions might indicate issues yet to develop symptomatically.
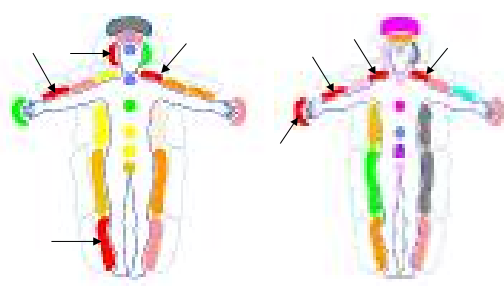

RFI chart of Case studies 3 (generally unhealthy) and 8 (generally healthy)

\section{CONCLUSIONS}

There is clear qualitative evidence from this study that these devices can go some way to identifying areas of imbalance. This gives argument for developing more integrated and non-invasive approaches of assessing health states.

Future studies should attempt to establish scanning protocols, which might ensure reliability and consistency whilst at the same time as allowing for the fact that these devices are detecting continually varying data at different levels and therefore leading to variations in their results.

Future research should explore the concurrent validity of each of the systems against already established measures of psychological and physiological health. However, at present the authors are unaware of such systems and hope that this study will act as a stimulus for further investigations in this area. It also seems important for benchmarks to be established by gathering data on 'healthy' case studies with whom known out of balance cases can be compared.

References

Bundzen, P., Korotkov, K., Unestahl, L.-E. (2002) Altered States of Consciousness: Review of Experimental Data Obtained with a Multiple Medicine, 8 (2), pp 153-167 Dobson, P. and Tchernychko, E. (2000) Investigations into Stress and it's Management using the Gas Discharge Visualisation Technique International 3 of Alternative and Complementary Medicine Jun Oschman, J., (2000) Energy Medicine: The Scientific Basis Churchill Livingstonc 